\title{
Psychiatric disorders and low Glasgow Coma Scale score - A case series
}

\author{
Muhammad Ajmal* \\ Locum anaesthetist, ID Medical, a locum agency, Milton Keynes, UK
}

\begin{abstract}
A low score on the Glasgow Coma Scale (GCS) [1], is a common presentation in acute hospital settings. Anesthesiologists and intensivists are frequently asked to ensure airway protection in such patients. Endotracheal intubation is necessary if the GCS is very low. However, it is important to confirm that consciousness is really impaired and the airway is unprotected because endotracheal intubation is demanding and not without risks. In a recent experience with two cases of low GCS, the plan for endotracheal intubation was unnecessary, as the patients were actually capable of protecting their airway.
\end{abstract}

\section{Case description}

1. A young woman with an apparent history of seizure disorder was taking antiepileptic medication. She presented to the emergency department with a persistently low GCS of $3 / 15$ after an episode of seizures. Her trachea was intubated and computed axial tomography (CT) of her brain was performed. The CT was normal. After some time, she coughed out the endotracheal tube and returned to a low GCS of 3/15. Further review of the case revealed a dissociative disorder and was suffering from pseudo--seizures.

2. A middle--aged woman with an apparent history of multiple sclerosis (MS), chronic obstructive pulmonary disease, chronic headache, stridor, eyesight problems, and cigarette smoking was already admitted to the hospital when her GCS suddenly dropped to 5/15. She had also a history of intensive care unit (ICU) admission for acute respiratory failure and was managed with artificial ventilation and tracheostomy. An endotracheal intubation was immediately planned to facilitate CT of her brain. An anesthesiologist incidentally noted that the patient clenched a suction--catheter with her teeth and did not allow suctioning her throat. With this observation, the plan for endotracheal intubation and CT was abandoned. Further review of the case revealed that MS was erroneously associated with her history for the past 30 years and that she frequently presents with a low GCS. Further assessment revealed that she was suffering from dissociative disorder.

\section{Discussion}

Head injury, spontaneous subarachnoid and intracerebral haemorrhage, ischaemic stroke, intracranial infection and brain abscess, general trauma, non-- traumatic coma, epilepsy and poisoning are the common organic causes of low GCS. Reliability of GCS varies from 0.85 to 0.32 [2]. Patients with certain psychiatric disorders can also present with apparently low GCS. In catatonic states, especially due to schizophrenia and mania, patients are conscious but can be mute and unable to move or can have unusual postures. In dissociative stupor, there is profound diminution or absence of voluntary movements and responsiveness to external stimuli, including painful ones. Although some degree of disturbance of consciousness may be present, the muscle tone, posture, breathing, and sometimes eye--opening and coordinated eye movements make it clear that the individual is neither asleep nor unconscious. The examination and investigation reveal no evidence of a physical cause, and there is positive evidence of psychogenic causation in the form of recent stressful events or problems [3]. It is important for anesthesiologists and intensivists to be aware of such conditions and be able to recognize them to avoid unnecessary endotracheal intubations and ICU admissions.

\section{References}

1. Teasdale G, Jennett B (1974) Assessment of coma and impaired consciousness. A practical scale. Lancet 2: 81-84. [Crossref]

2. Heard K, Bebarta VS (2004) Reliability of Glasgow Coma Scale for emergency department evaluation of poisoned patients. Hum Exp Toxicol 23:197-200.

3. World Health Organization (1992) Classification of mental and behavioural disorders The ICD-10. Geneva: WHO.
Copyright: (C2018 Ajmal M. This is an open-access article distributed under the terms of the Creative Commons Attribution License, which permits unrestricted use, distribution, and reproduction in any medium, provided the original author and source are credited.
${ }^{*}$ Correspondence to: Muhammad Ajmal, Locum anesthetist, ID Medical, a locum agency, Milton Keynes, UK, Tel: 00447575770303; E-mail: ajmal_c@hotmail.com Received: July 06, 2018; Accepted: July 31, 2018; Published: August 07, 2018 